\title{
Response to a behavioural treatment, biofeedback, in constipated patients is associated with improved gut transit and autonomic innervation
}

\author{
A V Emmanuel, M A Kamm
}

\begin{abstract}
Background-Although behavioural treatment (biofeedback) successfully treats the pelvic floor abnormalities in patients with idiopathic constipation, many patients also normalise their impaired bowel frequency. We postulated that a response may be associated with altered cerebral outflow via extrinsic autonomic nerves to the gut. We investigated whether treatment changes extrinsic innervation, using mucosal laser Doppler flowmetry, whether autonomic changes are gut specific, and whether it changes gut transit.

Materials and methods-Forty nine patients (44 female, mean age 39 years) with idiopathic constipation were studied before and after biofeedback treatment (mean five sessions). Rectal mucosal blood flow was measured by laser Doppler flowmetry to assess direct extrinsic gut nerve autonomic activity. To assess general autonomic activity, RR (interval between successive $R$ waves on the electrocardiogram) variability, Valsalva ratio, orthostatic adjustment ratio, and phase II:IV blood pressure ratio (II:IV) of the Valsalva manoeuvre were measured. All autonomic tests were compared with those of 26 healthy volunteers (19 female, mean age 37 years).
\end{abstract}

Results-Twenty nine of 49 patients were symptomatically improved. Treatment reduced those with $\leqslant 3$ bowel actions per week (27 $v 9$, pre $v$ post), need to strain (26 $v 9$ ), and laxative or suppository use (34 $v$ 9). Biofeedback reduced retained markers by $32 \%$ in those with slow transit and by $20 \%$ in those with normal transit. Twenty two had slow transit before treatment-14 felt symptomatic improvement of whom 13 developed normal transit. There was a significantly greater increase in rectal mucosal blood flow in patients who subjectively improved compared with those who did not $(29 \% v 7 \% ; p<0.03)$ and in those with improved bowel frequency (33\% $v$ 9\%, increased $v$ unchanged bowel frequency; $p<0.05)$. Thirty five patients had abnormal $R R$ variability and 33 an abnormal Valsalva ratio; one had an abnormal orthostatic adjustment ratio and one an abnormal II:IV ratio. None of the general cardiorespiratory autonomic reflexes was changed by treatment.
Conclusions-Biofeedback treatment affects more than the pelvic floor. Successful outcome after biofeedback treatment is associated with improved activity of the direct cerebral innervation to the gut and improved gut transit. This effect is gut specific; cardiovascular autonomic reflexes were not altered.

(Gut 2001;49:214-219)

Keywords: biofeedback; constipation; gut transit; autonomic innervation

Gut directed biofeedback retraining has become an established therapy for idiopathic constipation $^{1-5}$ with more than half of all adult patients responding. ${ }^{35}$ Biofeedback is a learning strategy based on operant conditioning. Although the main focus is on abdominal and pelvic coordination, patients with slow bowel transit may also respond, increasing their bowel frequency. ${ }^{3}$

There are several mechanisms by which improved large bowel function may occur as a result of this therapy. A change in pelvic floor contraction to pelvic relaxation during defecation may allow forward movement of large bowel contents. Alternatively, eliminating inappropriate pelvic floor contraction may prevent retrograde peristalsis. ${ }^{6-8} \mathrm{~A}$ third possibility is that this gut focused behavioural therapy alters cerebral efferent autonomic pathways which innervate the large bowel.

To investigate this last possibility would ideally involve the use of a technique which specifically measures the level of activity of efferent autonomic nerves innervating the gut. Previous studies have established that patients with chronic idiopathic constipation demonstrate abnormalities of vagal function, ${ }^{9-11}$ but such studies have employed cardiorespiratory and humoral tests. ${ }^{9-11}$ Cerebral efferent pathways however are known to be organ specific. ${ }^{12}$ The use of rectal mucosal blood flow measurement, using a laser Doppler technique as a means of assessing the level of activity of autonomic nerves directly innervating the gut, ${ }^{13}{ }^{14}$ has been previously developed and validated.

This prospective study was designed to assess whether gut transit is improved by biofeedback techniques in patients with idiopathic constipation. Secondly, it was intended to assess whether improved transit is associated with an alteration of the level of extrinsic autonomic nerve activity to the gut. Thirdly, it remained to be determined whether the effect

Abbreviations used in this paper: FU, flux units. 
of biofeedback was specific to the gut or if there was a more generalised effect on the level of autonomic activity, as measured by cardiorespiratory autonomic tests.

\section{Materials and methods \\ PATIENTS}

Forty nine consecutive adult patients with constipation referred to a tertiary centre (44 female; mean age 36 years, range 18-61) were studied. Nineteen of these patients had been previously studied. ${ }^{14}$ All patients had constipation as defined by the Rome II criteria, ${ }^{15}$ complaining of either decreased bowel frequency (less than three times per week), sensation of incomplete emptying or difficult evacuation on at least a quarter of occasions, or a need to strain on at least a quarter of occasions. This was not quantitated using a diary system; the subjective complaints were considered adequate for entry to the study, as a previous study in our unit had demonstrated an excellent correlation between patients' symptoms on interview and diary card observations. ${ }^{16}$ In a pharmacological study of treatment for constipation conducted in our unit, only two of 87 patients had diary data which did not correlate with their self reported symptoms. ${ }^{16}$ Organic and metabolic causes of constipation had been ruled out by previous endoscopy, barium enema, and blood testing. Patients did not have a large rectocoele and had not been operated on. All patients had a normal diameter colon. In the week prior to the first biofeedback session, patients were not allowed laxatives.

Details were obtained of the patient's bowel habits (frequency, consistency, need to strain or digitate), the presence of abdominal bloating, and the need for laxatives. This information was obtained both before and at the end of biofeedback treatment and recorded in the notes by the biofeedback therapist. Mean duration of symptoms for patients was 19 years (range 6-43). Of 49 patients, 27 had three or fewer bowel actions per week, 30 described their stool as hard or pellet-like, 26 needed to strain, and 19 anally digitated to achieve defecation. Thirty nine patients reported some degree of abdominal bloating. Thirty five patients were taking regular laxatives and nine were using suppositories or enemas.

Twenty six healthy volunteers (17 women; mean age 36 years, range 18-61) with no gastrointestinal symptoms were studied as controls for the purpose of autonomic and laser Doppler studies. They did not have biofeedback treatment.

PHYSIOLOGICAL ASSESSMENT

Before their first biofeedback session, patients underwent electromyographic assessment of external anal sphincter relaxation or contraction during simulated defecation.

A whole gut transit study, cardiorespiratory autonomic function testing, and rectal laser Doppler mucosal flowmetry were performed before the start of biofeedback treatment and again after the last treatment by the same investigator, who was blind to the patient's symptoms and treatment outcome.

\section{Whole gut transit}

Patients underwent two radio-opaque marker transit studies, according to previously published techniques, ${ }^{17}$ the first immediately before the first session of biofeedback and the second a mean of five months (range 3-8) after the end of treatment. Three sets of radiologically distinct markers were taken at 24 hour intervals and an abdominal $x$ ray taken 120 hours after ingestion of the first set. Retention of more than the normal range for any one of the three sets of markers was regarded as reflecting slow whole gut transit. ${ }^{17}$ Twenty two of 49 patients were identified as having slow transit and 27 had normal transit.

\section{Simulated defecation}

With the patient in the left lateral position, a lubricated balloon attached to a catheter was inserted into the rectum and inflated with $50 \mathrm{ml}$ of water. Two adhesive EMG electrodes were placed on the anal skin over the external anal sphincter. The patient was asked to expel the balloon and success was recorded as passage of the balloon within five minutes. A sustained increase in surface EMG activity ( $>50 \%$ increase from baseline) on attempted defecation was defined as inappropriate pelvic floor contraction. ${ }^{18}$ Fifteen patients could not expel the balloon (7/22 in the slow transit and $8 / 27$ in the normal transit group) and 32 had paradoxical anal sphincter contraction (13/22 in the slow transit and 19/27 in the normal transit group).

\section{Cardiorespiratory autonomic function testing}

Autonomic function tests were performed without knowledge of the patient's symptoms before and after biofeedback treatment. Vagal cholinergic function was measured using two methods. The interval between successive $R$ waves on the electrocardiogram (RR interval) in response to vagovagal reflex bradycardia induced by deep breathing was measured. Secondly, the peak to trough heart rate ratio during a Valsalva manoeuvre (that is "Valsalva ratio"), was measured. The Valsalva ratio is affected by sympathetic and parasympathetic function, although the latter predominates. Sympathetic adrenergic activity was assessed by the percentage change in resting pulse and systolic blood pressure in response to orthostatic tilt of the bed from horizontal to an angle of $40^{\circ} .{ }^{19}$ This is considered an adequate tilt to test sympathetic function. ${ }^{19}$ Secondly, systolic blood pressure in phase II (peak systolic blood pressure) and phase IV (trough systolic blood pressure) of the Valsalva manoeuvre were assessed and the "II:IV" ratio determined.

Laser Doppler studies of rectal mucosal blood flow Subjects were studied in a room maintained at $22^{\circ} \mathrm{C}$ after 15 minutes of acclimatisation and were examined in the left lateral position. After digital examination and rigid sigmoidoscopy had confirmed that the rectum was empty, the laser Doppler probe (DP6A; Moor Instruments, Axminster, UK) was introduced via the 
Table 1 Symptoms and transit results in all 49 patients before and after biofeedback, stratified according to slow transit

\begin{tabular}{|c|c|c|c|c|}
\hline & \multicolumn{2}{|c|}{ Slow transit $(n=22)$} & \multicolumn{2}{|c|}{ Normal transit $(n=27)$} \\
\hline & $\begin{array}{l}\text { Before } \\
\text { biofeedback }\end{array}$ & $\begin{array}{l}\text { After } \\
\text { biofeedback }\end{array}$ & $\begin{array}{l}\text { Before } \\
\text { biofeedback }\end{array}$ & $\begin{array}{l}\text { After } \\
\text { biofeedback }\end{array}$ \\
\hline Subjective improvement & - & 14 & - & 15 \\
\hline Bowel frequency $<3$ per week & 16 & 6 & 11 & 3 \\
\hline Hard or pellet stool & 15 & 4 & 15 & 3 \\
\hline Need to strain & 12 & 3 & 14 & 6 \\
\hline Need to digitate & 4 & 2 & 15 & 7 \\
\hline Abdominal bloating & 19 & 5 & 20 & 6 \\
\hline Laxative use & 20 & 5 & 14 & 4 \\
\hline Normal transit & - & 13 & 27 & 27 \\
\hline Mean number of retained markers & 42.5 & 32.4 & 17.1 & 15.2 \\
\hline
\end{tabular}

sigmoidoscope. Recordings were made at 10 $\mathrm{cm}$ from the anal verge at four points circumferentially at $90^{\circ}$ to each other. Readings were taken after the trace had stabilised for 30 seconds as previously described. ${ }^{13}$ Rectal mucosal blood flow measurements were performed without knowledge of the patient's symptoms (AVE) before and after biofeedback treatment. Results are presented as means (SD).

BIOFEEDBACK TREATMENT

All patients completed their treatment. Each patient was treated by one of two specialist nurse therapists for their entire course of treatment. Patients had a mean of five sessions (range 4-7) over a mean of 62 days (range 45-86). Each session lasted 30-60 minutes and patients were instructed in a variety of previously described techniques, ${ }^{35}{ }^{20}$ including feedback from an EMG trace where appropriate. Briefly, with the patient lying on their right side, facing the therapist and EMG display, a balloon is inserted into the rectum and inflated with 50 $\mathrm{ml}$ of air so that the patient has the sensation of needing to defecate. Adherent surface electrodes placed over the external anal sphincter allow the patient to see the EMG pattern made by voluntary sphincter contraction. The patient is then asked to expel the balloon and if there is an increase, rather than the normal decrease, in sphincter activity, the patient is taught how to strain without increasing sphincter activity. Patients are also taught how to strain effectively using bracing of the abdominal wall muscles and propulsion of pelvic floor musculature. Patients who digitate are instructed to stop doing so. They are given behavioural advice about frequency and length of toilet visits, posture on the toilet, and dietary habits. Laxative consumption is prohibited in all patients. Patients are instructed on basic gut anatomy and function, and an appreciation is also gained of psychological and social stresses which may be influencing the patient's functioning.

ETHICS APPROVAL

The study was given ethics approval by the ethics committee of St Mark's and Northwick Park Trust.

STATISTICAL ANALYSIS

Differences before and after treatment in each group were assessed by means of Fisher's exact two tailed test. Cardiorespiratory autonomic and laser Doppler mucosal flowmetric data were normally distributed. The two tailed $t$ test was used to compare the results of autonomic cardiorespiratory and rectal mucosal blood flow function tests before and after treatment.

\section{Results}

SYMPTOMS

At the end of treatment, 29 of 49 patients reported a subjective overall improvement in their symptoms.

Twenty seven patients before treatment and nine patients after treatment reported a bowel frequency of less than three per week. The mean number of stools per week in these 27 patients was 1.9 before treatment and this increased significantly to 3.9 after treatment $(\mathrm{p}<0.01)$. Thirty patients before treatment and seven after treatment reported their stools as being hard or pellet-like. Biofeedback treatment resulted in a reduction in the number of patients who needed to strain to defecate (from 26 to nine) and in the number of patients who needed to digitate to defecate (from 19 to nine). Biofeedback treatment relieved abdominal bloating in 28 of 39 patients who complained of this symptom.

Prior to starting biofeedback, 34 of 49 patients used regular oral laxatives (Senna, Lactulose, Fybogel, or Co-danthrusate), eight used regular glycerine suppositories, and one other used both a laxative and suppositories to achieve defecation. Following treatment nine patients were in need of laxatives and none used suppositories; of the nine still consuming laxatives two had reduced the dose by at least half.

\section{PHYSIOLOGICAL INVESTIGATIONS}

\section{Whole gut transit}

Before biofeedback. Twenty two of 49 constipated patients were identified as having slow transit. Of these 22, seven had marker retention predominantly in the rectosigmoid ${ }^{21}$ as defined by more than half of the excessively retained markers being present in the rectosigmoid; the remaining 15 patients with slow transit had excessive marker retention throughout the colon.

After biofeedback. Fourteen of 22 slow transit constipation patients reported global subjective improvement after biofeedback; 15 of 27 patients with normal transit reported a similar improvement; the difference between the two groups was not significant $(\mathrm{p}=0.77)$. Similarly, there was no difference between patients with slow and normal transit for the proportion who improved in relation to the symptoms of straining, bloating, digitation, and laxative use following biofeedback treatment (table 1).

Among 22 patients with slow transit, four of seven with only rectosigmoid delay and 10 of 15 in whom slow transit was due to a more generalised hold up of markers, reported subjective overall improvement; the difference between the two groups was not significant $(\mathrm{p}=1.00)$.

At the end of treatment, 14 of 22 patients with slow transit felt subjectively improved and 12 had increased their bowel frequency; 13 of 
these 14, but none without symptomatic improvement, had normalised their whole gut transit. All of these 13 patients felt subjective overall improvement and 12 had objectively increased their bowel frequency. When considering the 14 patients with slow transit who reported subjective improvement, there was a reduction in the mean number of retained markers by $32 \%$ (42.3 $v 28.8$ retained out of 60 , pre $v$ post treatment; $p<0.01)$. Of the eight with slow transit who did not feel subjectively improved, the number of retained markers fell by $9 \%$ (43.5 $v 39.6$ retained out of 60 , pre $v$ post treatment; $\mathrm{p}=0.22$ )

Of the 15 patients with normal transit before treatment who reported subjective improvement, there was a reduction in the mean number of retained markers of $20 \%$ (17.4 v 13.9 retained out of 60 , pre $v$ post treatment; $\mathrm{p}<0.05)$. When considering the 12 with normal transit who did not report improvement after biofeedback, there was a non-significant change in retained markers of 8\% (16.8 $v 18.1$ retained out of 60 , pre $v$ post treatment; $\mathrm{p}=0.14$ ).

\section{Simulated defecation}

Paradoxical contraction of the external anal sphincter was present in 35 of 49 constipated patients before biofeedback treatment. This was still present after treatment in one patient. Twenty two of 35 patients with and seven of 14 patients without paradoxical contraction before treatment reported subjective improvement after biofeedback $(p=0.52)$. Again, there was no difference between patients with and without paradoxical contraction for any of the other outcome measures.

Laser Doppler studies of rectal mucosal blood flow Before biofeedback. As has been demonstrated previously, ${ }^{14}$ patients with constipation had reduced mean rectal mucosal blood flow compared with age and sex matched controls (156.5 (9.2) v 186.0 (13.7) flux units (FU), median (SD)). This reduction in mucosal blood flow was most marked in those with slow transit constipation (140.0 (7.8) FU $v 163.5$ (11.1) FU, slow $v$ normal transit; $\mathrm{p}=0.034$ ) while there was no difference in mucosal blood flow between those with or without paradoxical contraction (152.2 (11.6) FU $v 154.2$ (8.5) FU, paradoxical contraction present $v$ paradoxical contraction absent; $\mathrm{p}=0.38$ ).

Following biofeedback. Rectal mucosal blood flow increased significantly in all 29 subjects who felt subjectively better after biofeedback (fig 1). The mean increase for these subjects was from 151.4 (10.3) FU to 194.8 (16.9) FU, a mean increment of $28.7 \%$; this was significantly greater than the mean increment of $6.5 \%$ observed in those subjects reporting no subjective improvement $(\mathrm{p}<0.03)$. Similar changes in rectal mucosal blood flow were evident when examining objective markers of improvement in constipation-increased bowel frequency, reduction or cessation of laxative use (fig 1), and subjective reduction of bloating.

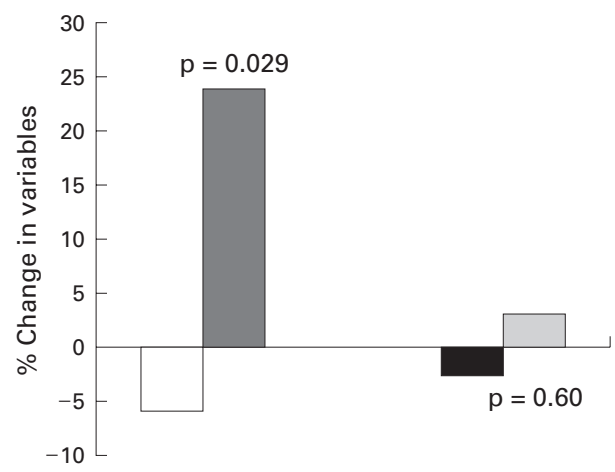

$\square$ Rectal mucosal blood flow in patients who continued to use laxatives "failed treatment"

$\square$ Rectal mucosal blood flow in patients who stopped using laxatives "successful treatment"

aR variation in patients who continued to use laxatives "failed treatment"

$\square R R$ variation in patients who stopped laxatives "successful treatment"

Figure 1 An increase in rectal mucosal blood flow was observed in those subjects who reduced or stopped laxative use ("responders" or "successful treatment") after biofeedback compared with those who did not ("non-responders" or "failed treatment") $(p=0.029)$. No such alteration in RR interval variability was noted. This suggests that response to treatment was associated with specifically improved autonomic innervation to the gut, without change in cardiovascular autonomic dysfunction.

There were no significant alterations in mucosal blood flow for those who reported a reduction in the need to strain or digitate to achieve defecation.

There was no difference in the degree of increase in rectal mucosal blood flow between those with slow and normal transit pretreatment $(30.3 \% v 27.8 \%$, respectively; $\mathrm{p}=0.20)$ and those with or without paradoxical contraction pretreatment $(27.0 \% \vee 30.3 \%$, respectively; $\mathrm{p}=0.16$ ).

Correlation of blood flow with alteration of transit is shown in fig 2 . There was a significant correlation between change in rectal mucosal blood flow and change in number of retained markers $(r=-0.55, \mathrm{p}=0.043)$.

Cardiorespiratory autonomic function testing Before biofeedback. In comparison with controls, patients with constipation had attenuation of

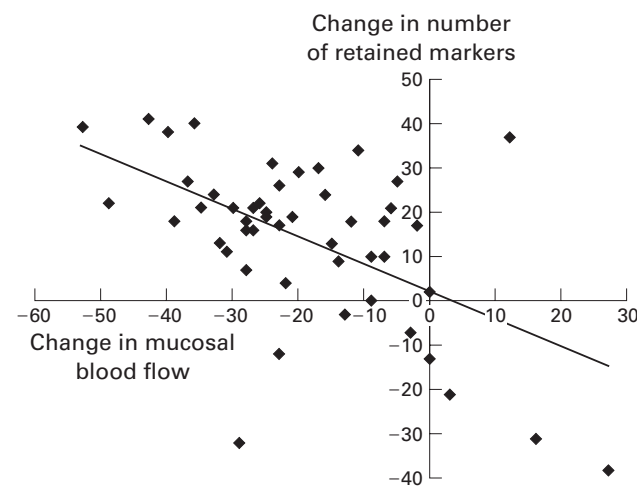

Figure 2 Correlation between change in transit (reduction of retained markers) and change in extrinsic gut innervation (increased rectal mucosal blood flow) in patients after behavioural treatment (biofeedback) $(r=-0.55, p=0.043)$. 
their cardiovagal cholinergic tests, namely RR interval variability and Valsalva ratio. Of the 49 constipated patients, RR interval variability was outside the normal range in 35 $(p<0.00001)$ and the Valsalva ratio was abnormal in $33(\mathrm{p}<0.0001)$ compared with two abnormal results for both tests in controls.

This abnormality was most marked in 22 patients with slow transit compared with those with normal transit, 21 of whom had abnormal $R R$ interval variability $(p=0.013)$ and 20 had an abnormal Valsalva ratio $(\mathrm{p}=0.08)$. In contrast, tests of sympathetic adrenergic activity were not significantly different from controls, with three of 49 subjects (two slow transit) having an abnormal orthostatic adjustment ratio and four of 49 (two slow transit) an abnormal II:IV ratio.

Following biofeedback. Neither the predominantly vagal cholinergic tests ( $R R$ variabilitytwo patients normalised; Valsalva ratio-one normalised, two becoming more abnormal) nor sympathetic adrenergic tests (orthostatic adjustment ratio-no alterations; II:IV ratioone normalised) were significantly affected by biofeedback treatment (fig 1).

\section{Discussion}

This prospective study has shown that gut directed biofeedback is an effective behavioural treatment for chronic idiopathic constipation, with $59 \%$ of patients reporting subjective improvement. This improvement was seen in patients with slow transit $(63 \%)$ and normal transit $(56 \%)$. Patients in this study did not have bowel function quantitated using a diary system although transit time was formally assessed in all patients. Subjective complaints were considered adequate for entry to the study in those patients in whom measured transit was normal, as we have previously shown that this correlates well with a diary card record. ${ }^{16}$ Normal subjects completing a transit study will have passed more than $80 \%$ of markers at 120 hours. ${ }^{17}$ The use of three different shaped markers taken on consecutive days reduces the effect of day to day variation in transit time. It was thus possible to characterise the group with slow transit in a reproducible and validated fashion. Accompanied by the subjective improvement were objective improvements of transit, with $59 \%$ of slow transit patients normalising their transit by the end of treatment. This study has also shown that the change in transit occurred in patients with excessive retained markers distributed around the colon. The effect is therefore one which may relate to whole colon function or innervation, and not just the distal large bowel.

Treatment also significantly hastened transit in those with normal transit pretreatment, with an $18 \%$ reduction in the number of markers present on the follow up transit study.

The mechanism of action of biofeedback treatment is more complex than just an effect on the pelvic floor-it was as effective in patients with slow transit as it was in those with paradoxical contraction, with $63 \%$ of those with paradoxical contraction and $50 \%$ of those without paradoxical contraction reporting subjective improvement after treatment. Previous studies have shown that patients with and without anismus, with both slow and normal transit, males and females, all derive benefit equally from biofeedback. ${ }^{35}$

There are several mechanisms by which behavioural treatment may have altered gut function and blood flow. Cerebral autonomic control of the gut and its microcirculation may have been changed. Alternatively, it is possible that the observed increases in rectal mucosal blood flow were due to improvement in psychological or social functioning brought about by behavioural treatment. We have previously shown that the level of activity of extrinsic autonomic innervation correlates with physiological parameters. ${ }^{22}$

Since it has been previously shown that constipated patients, in comparison with controls, have a selective deficit of cardiovagal cholinergic activity, ${ }^{9} 1023$ we hypothesised that improvements, both subjectively and in transit, following biofeedback may be accompanied by improvements in the level of extrinsic autonomic activity. In previous studies we have shown that constipated patients have a reduction in rectal mucosal blood flow, an index of the level of activity of extrinsic autonomic innervation to the gut. ${ }^{1314}$ This reduction in blood flow is most marked in those with slow transit, suggesting that these patients form a distinct subgroup of the population of constipated patients, with distinct physiological differences from those with normal transit.

This study confirmed the findings of others $^{910}$ that patients with constipation, compared with healthy controls, had significantly abnormal cardiovagal cholinergic test scores. It has also been demonstrated that they have reduced rectal blood flow. These abnormalities were most marked in those with slow transit. It has also been shown that the reduction in microcirculatory flow is strongly correlated with parasympathetic cholinergic dysfunction. Successful outcome after biofeedback, assessed both subjectively and objectively, was associated with an increase in rectal mucosal blood flow but no alteration of abnormal cardiovascular autonomic reflexes. This would suggest that the improvement in the level of autonomic activity is not a generalised phenomenon but rather one specific to gut innervation. A similar specificity of response has been described for biofeedback applied to hypertension ${ }^{24}$ and tension headache. ${ }^{25}$ Cardiovascular vagal innervation therefore appears to be regulated, at least partly, independently of gut vagal innervation. Constipated patients continued to demonstrate cardiovascular vagal dysfunction after improvement in gut function, suggesting the presence of a more generalised vagal abnormality or the presence of other factors such as anxiety. The results of this study provide evidence for diminished cholinergic innervation affecting both colonic transit and mucosal blood flow in patients with slow transit. We did not find evidence of excessive sympathetic inhibitory innervation on cardiorespiratory testing. The improvement in gut blood flow is at least in 
part likely to be due to the balance of cholinergic and sympathetic innervation, both of which are thought to influence motor control of the gut microcirculation. ${ }^{26}{ }^{27}$ As the sympathetic system is predominantly inhibitory to gut transit, ${ }^{26-28}$ decreased sympathetic inhibition or enhanced cholinergic activity were likely to have resulted in increased transit and increased microcirculatory flow. As cardiorespiratory reflexes were not altered, and mucosal blood flow does not distinguish between these influences, we cannot be precise about which of these changes was more important. A previous study has shown that rectal mucosal flux also correlated strongly with whole gut transit, as measured using radio-opaque markers. ${ }^{14}$ The slower the transit the more profound was the reduction in mucosal flow.

The parallel changes in motility (transit) and blood flow that follow successful response to biofeedback suggest that they may result as a direct consequence of changes in the level of extrinsic autonomic efferent activity to the gut. Changes in blood flow should not be thought of as occurring purely secondary to motility changes since it is known that alterations in gut blood flow, secretion, and motility occur simultaneously in acute human experiments. ${ }^{29-31}$ However, a change in mucosal blood flow secondary to altered motor function cannot be excluded. It may be possible to study this question by examining the mucosal blood flow response to an increase in transit produced by laxatives.

The fact that there was no demonstrable change in extraintestinal measures of autonomic function, such as cardiorespiratory autonomic tests, suggests that this amelioration of autonomic activity is gut specific. The implications of this observation are that in some patients the changes in rectal mucosal blood flow and presumably motility are secondary to changeable extrinsic neural function rather than permanent damage to the intrinsic enteric neurones.

In conclusion, successful response to behavioural treatment (biofeedback) for constipation is associated with specifically improved autonomic innervation to the large bowel and improved transit time for patients with slow and normal transit.

We are indebted to Julie Storrie, Nicky Horton, and Jaqui Bowers for assistance in performing the biofeedback treatment. The statistical advice of Caroline Dore is also gratefully acknowledged.

1 Enck P. Biofeedback training in disordered defecation. A critical review. Dig Dis Sci 1993;38:1953-60.

2 Bennings MA, Buller HA, Imainiau JAJM. Biofeedback training in chronic constipation. Arch Dis Child 1993;68: $126-9$
3 Koutsomanis D, Lennard-Jones JE, Kamm MA. Prospective study of biofeedback treatment for patients with slow and normal transit constipation. Eur $\mathcal{f}$ Gastroenterol Hepatol 1994;6:131-7.

4 Bassotti G, Whitehead WE. Biofeedback as a treatment approach to gastrointestinal tract disease tract disorders. Am $\mathcal{F}$ Gastroenterol 1994;89:2-7.

5 Chiotakakou-Faliakou E, Kamm MA, Roy AJ, et al. Biofeedback provides long term benefit for patients with intractable slow and normal transit constipation. Gut 1998;42:517-21.

6 Halls J. Bowel content shift during normal defaecation. Proc $R$ Soc Med 1965;58:859-60.

7 Crowell MD, Bassotti G, Cheskin LJ, et al. Method for prolonged ambulatory monitoring of high amplitude propagated contraction from the colon. Am f Physiol 1991;261: G263-8.

8 Herbst F, Kamm MA, Morris GP, et al. Gastrointestinal transit and prolonged ambulatory colonic motility in health and faecal incontinence. Gut 1997;41:381-9.

9 Altomare D, Pilot MA, Scott M, et al. Detection of a subclinical autonomic neuropathy in constipated patients using a sweat test. Gut 1992;33:1539-43.

10 Aggarwal A, Cutts TF, Abell TL, et al. Predominant symptoms in irritable bowel syndrome correlate with specific autonomic nervous system abnormalities. Gastroenterology 1994;106:945-50

11 Jorgensen LS, Christiansen P, Raundahl U, et al. Autonomic nervous system function in patients with functional abdominal pain. An experimental study. Scand f Gastroenterol 1993;28:63-8.

12 Livingston RB. Visceral control mechanisms. In: West JB, ed. Best and Taylor's physiological basis of medical practice, 11 th edn. Baltimore: Williams and Wilkins, 1988:64-74.

13 Emmanuel AV, Kamm MA. Laser Doppler measurement of rectal mucosal blood flow. Gut 1999;45:64-9.

14 Emmanuel AV, Kamm MA. Laser Doppler flowmetry as a measure of extrinsic colonic innervation in functional bowel disease. Gut 2000;46:212-17.

15 Thompson WG, Longstreth GF, Drossman DA, et al. Functional bowel disorders and functional abdominal pain. Gut 1999;45(supp II):I143-7.

16 Emmanuel AV, Nicholls TJ, Roy AJ, et al. Prucalopride improves colonic transit and stool frequency in patients with slow and normal transit constipation. Gastroenterology 2000;118(suppl 2):4438.

17 Evans RC, Kamm MA, Hinton JM, et al. The normal range and a simple diagram for recording whole gut transit time. Int $\mathcal{F}$ Colorectal Dis 1992;7:15-17.

18 Preston DM, Lennard-Jones JE. Anismus in chronic constipation. Dig Dis Sci 1985;30:413-18.

19 Ewing DJ. Cardiovascular reflexes and autonomic neuropathy. Clin Sci Med 1978;55:321-7.

20 Koutsomanis D, Lennard-Jones JE, Roy AJ, et al. Controlled, randomised trial of visual biofeedback versus muscle training without a visual display for intractable constipation. Gut 1995;37:95-9.

21 Arhan P, Devroede G, Jehannin B, et al. Segmental colonic transit time. Dis Colon Rectum 1981;24:625-9.

22 Emmanuel AV, Kamm MA, Mason HJ. Direct evidence of psychological state affecting extrinsic innervation of the gut-laser Doppler flowmetry to measure mucosal blood flow. Gastroenterology 1997;112(suppl):A728.

23 Fukudo S, Nomura T, Muranaka M, et al. Brain-gut response to stress and cholinergic stimulation in irritable bowel syndrome. f Clin Gastroenterol 1993;17:133-41.

24 Schwartz GE. Biofeedback, self-regulation and the patterning of physiological processes. Am Sci 1975;63:314-24.

25 Tarler-Benlolo. Role of relaxation in biofeedback training. Psychol Bull 1978;85:727-55.

26 Vanner S, Surprenant A. Neural reflexes controlling intestinal microcirculation. Am f Physiol 1996;271:G223-30.

27 Thiefin G, Raybould HE, Leung FW, et al. Capsaicinsensitive afferent fibres contribute to gastric mucosal blood flow response to electrical vagal stimulation. Am f Physiol 1990;259:G1037-43.

28 McIntyre AS, Thompson DG. Adrenergic control of motor and secretory function in the gastrointestinal tract. Aliment Pharmacol Ther 1992;6:125-42.

29 Beaumont W. Experiments and observations on the gastric juice and the pathophysiology of digestion. Plattsburg, New York: FP Allen, 1897

30 Wolf S, Wolf HG. Experimental study of changes in gastric function in response to varying life experiences. Rev Gastroenterol 1947;14:419-26. ?? J title not right

31 Almy TP. Experimental studies on the irritable colon. Am $\mathcal{f}$ Med 1951;10:60-7. 